\title{
Performance of Hypotension Prediction Index for Prevention of Hypotension and Results on Biomarkers of Organ Injury and Oxidative Stress During Elective Non-Cardiac Surgery: A Randomized Controlled Trial
}

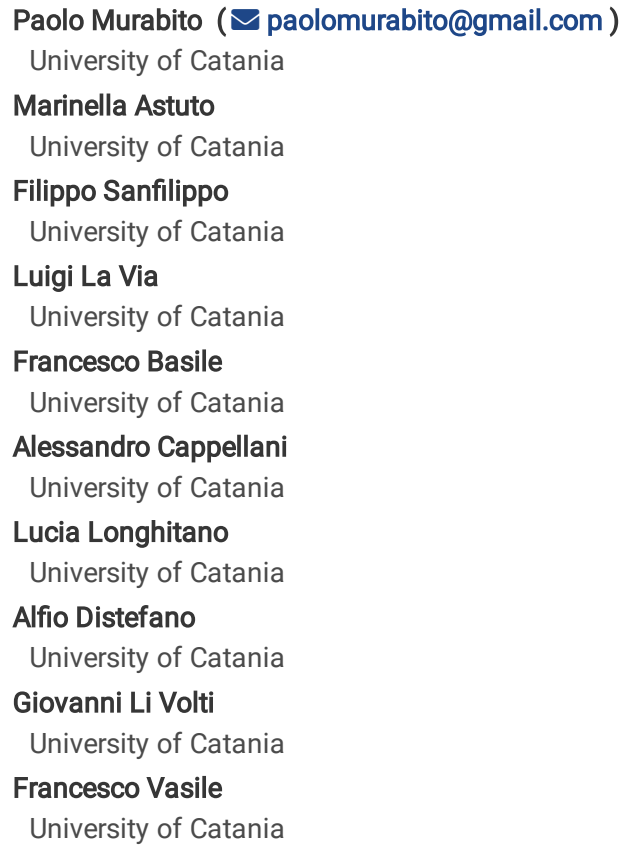




\section{Abstract}

Background: Intraoperative hypotension is associated with increased postoperative morbidity and mortality. We tested whether the clinical application of an early warning system (EWS) in combination with an algorithm for hemodynamic management reduces the occurrence of intraoperative hypotension as well as decrease the degree of organ injury and oxidative stress.

Methods: We randomly assigned patients undergoing major general surgery EWS and hemodynamic algorithm (intervention group, $\mathrm{n}=20$ ) or standard care $(n=20)$. The primary outcome was the difference in hypotension (defined as mean arterial pressure $<65 \mathrm{mmHg}$ ) evaluated as episodes, time and time-weighted average of hypotension. As secondary outcome we assessed surrogate markers of organ injury (neuron specific enolase -NSE), S100B protein, high-sensitive troponin, neutrophil gelatinase-associated lipocalin -NGAL) and oxidative stress (reduced glutathione).

Results: The median number of hypotensive episodes was lower in the intervention group [-5.0 (95\%Cl:-9.0,-0.5); $\mathrm{P}<0.005]$, with lower time spent in hypotension [-12.8 minutes (95\%Cl:-38.0,-2.3 min); $\mathrm{P}<0.001]$, correspondent to $-4.8 \%$ of total surgery time $(95 \% \mathrm{Cl}:-12.7,0.01 \%$; $\mathrm{P}<0.05)$. The median time-weighted average of hypotension was $0.12 \mathrm{mmHg}(0.35)$ in the intervention group and $0.37 \mathrm{mmHg}(1.11)$ in the control group, with a median difference of $-0.25 \mathrm{mmHg}$ $(95 \% \mathrm{Cl}:-0.85,-0.01 ; \mathrm{P}<0.05)$. NGAL was correlated with time-weighted average of hypotension $(\mathrm{R}=0.32 ; \mathrm{P}<0.05)$ and $\mathrm{S} 100 \mathrm{~B}$ with all primary outcomes investigated (all $\mathrm{P}<0.001$ ). The intervention group had lower NSE and higher reduced glutathione when compared to the control group.

Conclusions: The use of an EWS coupled with an hemodynamic algorithm resulted in reduced intraoperative hypotension. This finding was coupled with a reduction of biomarkers of brain injury and oxidative stress.

Trial Registration number and date: "Correlation Between Circulating Biomarkers of Organ Damage and Intraoperative Hypotension Management", NCT03527758, registered on May 17, 2018.

https://www.clinicaltrials.gov/ct2/show/NCT03527758?term=NCT03527758\&draw=2\&rank=1

\section{Background}

Hemodynamic instability represents a relatively common clinical event during surgical procedures. In particular, intraoperative hypotension is common and may cause organ ischemia, finally leading to injury and increasing the risk of postoperative complications. In this regard, several studies reported a significant correlation between intraoperative hypotension and organ injury[1-4]. Although the definition of intraoperative hypotension is variable, it is most commonly defined in terms of absolute systolic or mean arterial pressure (MAP) reduction, or relative systolic or MAP reduction from baseline values[5].

There is evidence that the risk of harm is due not only the severity (time and/or duration) of a single event but also to a series of shorter hypotensive episodes; therefore, even relatively short but multiple episodes of hypotension may worsen patient's outcome. In this context, the key-player leading to organ injury appears to be the mismatch between oxygen supply and demand[6-8]. Furthermore, after periods of ischemia, tissue reperfusion may lead to increased production of oxidative stress (i.e. reactive oxygen species, isoprostanes, lipid hydroperoxides) concurring to organ injury. However, the characterization and definition of intraoperative hypotension remains debated and vague. In a systematic review, Bijker et al.[5] found 140 definitions for hypotension reported by 130 articles. As consequence, the described incidence of intraoperative hypotension ranged from $5-99 \%[5,9]$. Nevertheless, there is a wide agreement regarding the importance of promptly treating intraoperative hypotension with the aim of preventing postoperative complications. Previous studies evaluated if hypotensive events could be predicted allowing clinicians to move from a reactive to a proactive approach, avoiding or reducing the impact of intraoperative hypotension. A recent randomized trial showed that the use of a machine learning-derived early warning system (EWS) as compared with standard care resulted in significantly lower intraoperative hypotension[10]. However, no studies investigated whether such proactive approach is coupled with a reduced organ injury as measured by biomarker assessment. Therefore, we aimed at evaluating the impact of an EWS with an algorithm for hemodynamic management on the intraoperative time spent with hypotension in adult patients undergoing major general surgery; we also assessed the impact of the intervention on post-operative levels of biomarkers of organ injury and oxidative stress.

\section{Materials And Methods}

The present single center randomized clinical trial took place at the "Azienda Ospedaliera Universitaria Policlinico-San Marco, site Gaspare Rodolico", a tertiary academic center. The study was approved by the local institutional ethics committee "Catania 1 Ethical Committee of Policlinico - San Marco University Hospital" - reference protocol number: NL62115.018.17. The study was registered on www.clinicaltrialgov (NCT03527758) on 17/05/2018.

\section{Patients}

We randomized adult patients ( $\geq 18$ years old) undergoing elective laparotomic major general surgery under general anesthesia if an intraoperative continuous invasive blood pressure monitoring was planned. Written informed consent was obtained from all patients before surgery. The first participant was enrolled in January 2019, and the last follow-up was in January 2020. A phone interview was performed 30 days following surgery in order to record the onset of late postsurgical complications and/or hospital readmissions.

A target MAP of at least $65 \mathrm{mmHg}$ during surgery was mandatory. Patients for whom the attending anesthesiologists requested a different MAP target were excluded. Other exclusion criteria were: emergency surgery, patients with cardiac failure or cardiac shunts, severe aortic stenosis, or preoperative cardiac arrhythmias (in accordance with the summary of product characteristics of the EWS), hypotension (MAP<65 mmHg) before surgery, acute or chronic renal disease, need for vascular clamping during surgical procedure. The study protocol and statistical analysis plan are available in Supplementary Digital Content 1 and 2 . 


\section{Randomization}

Patients were randomized into two different groups ( $\mathrm{n}=20$ each): the intraoperative EWS monitoring coupled with an algorithm for hemodynamic management (intervention group) or standard care (control group). A computer-generated permutated block randomization (concealed and varying permutated block sizes of 4 and 6 patients) was used with a 1:1 allocation ratio. The researchers involved in the study for laboratory measurements and statistical analyses were blinded to group allocation.

\section{Procedure}

A radial artery catheter was placed in all patients prior to the induction of general anesthesia and it was connected to the FloTrac IQ sensor with EWS software in the intervention group, and to standard FloTrac sensor (both marketed by Edwards Lifesciences ${ }^{\circledR}$ ) in the control group. System zeroing and quality control of the arterial signal was performed by the anesthesiologist. The arterial pressure waveform was measured continuously with a sample frequency of $100 \mathrm{~Hz}$. The standard FloTrac and the FloTrac IQ pressure transducers were connected to the HemoSphere monitor (Edwards Lifesciences ${ }^{\circledR}$ hereafter referred to as the advanced hemodynamic monitor), and the resulting electrical signal was transmitted to a Mindray ${ }^{\circledR}$ monitor (hereafter referred to as the standard monitor). The HemoSphere monitor displayed commonly used hemodynamic parameters (Stroke Volume, Stroke Volume Variation, Cardiac Index, Systemic Vascular Resistance) calculated from the waveform every 20 seconds, as well as the Hypotension Prediction Index (HPI) (updated every 20 seconds). The standard monitor displayed the MAP, systolic arterial pressure and diastolic arterial pressure. In the control group, the advanced hemodynamic monitor was connected in order to collect the intraoperative data, but the screen was fully covered and the alarms were silenced; anesthesiologists solely used the variables visible on the standard monitor (i.e. invasive blood pressure) to guide hemodynamic treatment.

Upon reaching the predefined MAP threshold $(<65 \mathrm{mmHg})$, the anesthesiologist treated the patient in accordance with a standardized algorithm in use at our Hospital. In the intervention group, when the HPI value was greater than $85 \%$, the anesthesiologists applied a proactive algorithm for hemodynamic management after a check for artifacts; (Figure 1) the algorithm was based on the values of advanced hemodynamic parameters (Stroke Volume Variation, Dynamic Arterial Elastance, Maximum Delta Pressure over Delta Time). In both groups, each pharmacological intervention was recorded by an external observer. At the end of the procedure, all the data were downloaded from the advanced hemodynamic monitor.

\section{Outcomes}

Our primary outcome was the evaluation of hypotension (defined as MAP $<65 \mathrm{mmHg}$ ) in the two groups. This outcome was described by four variables:

- number of hypotensive events,

- absolute time spent with hypotension during surgery (minutes),

- time spent in hypotension relative to surgical duration (\%),

- time-weighted average of hypotension during surgery, measured by calculating the area under the threshold divided by the total duration of surgery. Practically, this parameter is calculated as the maximum depth of hypotension below the threshold of MAP<65 mmHg (unit: $\mathrm{mmHg}$ ) $\times$ total time spent in hypotension (unit: minutes) divided by total duration of surgery (unit: minutes). In example, a patient undergoing surgery lasting 100 minutes experiences 5 episodes of hypotension, all of them lasting 1 minute and all with a minimal MAP of $60 \mathrm{mmHg}$. In this case, the area under the threshold is $25 \mathrm{mmHg}$ per minute (calculated as 5 minutes of hypotension $\times 5 \mathrm{mmHg}$ of MAP $<65 \mathrm{mmHg}$ ). Finally, the time-weighted average will be $25 \mathrm{mmHg}$ per minute divided by 100 minutes of surgery, corresponding to $0.25 \mathrm{mmHg}$.

The secondary outcome was to compare the levels of biomarkers of brain, myocardial and kidney injury and oxidative stress in both groups. Blood samples in this regards were collected at three time-points from the radial artery: prior to the induction of general anesthesia (T0), 2 hours after (T1) and at the end of the surgical procedure (T2). Samples were allowed to clot for 30 minutes at room temperature before centrifugation for 15 minutes at $1000 \times \mathrm{g}$. Serum was then immediately separated in aliquots and stored at $-80^{\circ} \mathrm{C}$ to avoid repeated freeze-thaw cycles. A blood gas analysis was immediately performed after blood sample collection. For the purposes of this first preliminary study, only the samples obtained at T0 and T2 were used.

The following biomarkers of brain, myocardial and kidney injury, as well as oxidative stress were evaluated in both groups: neuron-specific enolase (NSE), S100B protein, high-sensitive troponin (hs-TPN), neutrophil gelatinase-associated lipocalin (nGAL), hypoxia-inducible factor-1 alpha (HIF-1alpha), acetyl-CoA reduced glutathione (GSH), lipid hydroperoxide $(\mathrm{LOOH})$. The biochemical procedures for the sampling, storage and measurement of these biomarkers is provided as Supplementary Digital Content 3.

\section{Statistical Analysis}

Based on previously published studies $[1,3,10]$ and an expected reduction of incidence of hypotension from $80-38 \%$, it was calculated that a sample size of 40 patients, 20 in each group, would have $80 \%$ power to detect this effect using a 2-group $t$ test with an a $=0.05$, at 2-sided significance level.

SPSS version $17^{\circledR}$ was used to perform the statistical analysis. Categorical data are presented as frequencies and percentages. Differences between categorical data were analyzed using the Chi-Square test. Distribution of values was tested for normality with Kolmogorov-Smirnov test. Continuous data are presented as medians with interquartile range (IQR). The difference of hypotension-related outcomes, as well as the pre- post-surgery variation of biomarkers of inflammation and hypoxia between intervention and control group are presented as median differences and $95 \%$ confidence intervals (Cls) calculated with the Hodges-Lehmann method. The differences between continuous data were analyzed using the Mann-Whitney Utest. The Spearman's correlation coefficients were calculated to investigate the relation between biochemical markers of inflammation and hypotension-related outcomes. For each of the analyses, a 2 -tail probability value of $P<0.05$ was considered to be statistically significant.

Page $3 / 9$ 


\section{Results}

\section{Study population}

As shown in Table 1, all the baseline characteristics did not differ between groups. In particular, the majority of patients underwent gastrointestinal surgery and no differences in surgery duration was observed between groups.

Table 1

Background characteristics of the intervention and control groups.

\begin{tabular}{|llll|}
\hline & Intervention & Controls & p-value \\
\hline Age (years, median) & 69.0 & 70.5 & 0.39 \\
BMI, (Kg/m ${ }^{2}$, median) & 25.3 & 25.6 & 0.15 \\
Gender, $\mathrm{n}(\%)$ & & & \\
Men & $10(50 \%)$ & $12(60 \%)$ & 0.62 \\
Women & $10(50 \%)$ & $8(40 \%)$ & \\
ASA classification, $\mathrm{n}(\%)$ & & & \\
I & $1(5 \%)$ & $1(5 \%)$ & 0.94 \\
II & $8(40 \%)$ & $9(45 \%)$ & \\
III & $11(55 \%)$ & $10(50 \%)$ & \\
\hline Type of surgery, $\mathrm{n}(\%)$ & & & 0.81 \\
Gastrointestinal & $18(90 \%)$ & $16(80 \%)$ & \\
Gynecological & $1(5 \%)$ & $2(10 \%)$ & 0.18 \\
Others* & $1(5 \%)$ & $2(10 \%)$ & \\
Comorbidities, $\mathrm{n}(\%)$ & & & 0.22 \\
Hypertension & $10(50 \%)$ & $14(67 \%)$ & \\
Type-2 diabetes & $2(10 \%)$ & $4(19 \%)$ & 0.35 \\
Others & $8(40 \%)$ & $7(33 \%)$ & 0.49 \\
\hline Surgery duration, min (median) & $207.0(64.0)$ & $237.0(121.0)$ & 0.18 \\
\hline
\end{tabular}

ASA: American Society of Anesthesiologist. BMI: Body Mass Index. * included nephrectomy and removal of abdominal liposarcoma

\section{Hypotension-related outcomes}

The intervention group had lower number of hypotensive events than the control group [3 (IQR 6) vs. 8 (IQR 8) median times over surgery time, respectively], with lower time of surgery spent in hypotension (both absolute time and time relative to surgical duration, Table 2). The median difference of the incidence of hypotensive episodes was -5.0 episodes $(95 \% \mathrm{Cl}-9.0,-0.5 ; \mathrm{P}<0.001)$, with a median difference of total time spent in hypotension of -12.8 minutes $(95 \% \mathrm{Cl}-38.0$, $-2.3 \mathrm{~min} ; \mathrm{P}<0.001)$, correspondent to $-4.8 \%$ of total surgery time $(95 \% \mathrm{Cl}-12.7,0.01 \% ; \mathrm{P}<0.05)$ (Table 2$)$.

Table 2

Comparison of hypotension-related outcomes in the intervention and control group.

\begin{tabular}{|c|c|c|c|c|c|c|}
\hline & \multicolumn{2}{|l|}{ Median (IQR) } & \multirow[t]{2}{*}{ Median difference } & \multicolumn{2}{|l|}{$95 \% \mathrm{Cl}$} & \multirow[t]{2}{*}{$P$ value } \\
\hline & & & & Lower & Higher & \\
\hline & Intervention & Controls & & & & \\
\hline Number of hypotensive episodes, $\mathrm{n}$ & $3(6)$ & $8(13)$ & -5.0 & -9.0 & $-0.5^{\star \star}$ & $<0.001$ \\
\hline Total time spent in hypotension, $\min$ & $4.3(11)$ & $21.3(28)$ & -12.8 & -38.0 & $-2.3^{\star \star}$ & $<0.001$ \\
\hline Time in hypotension relative to surgical duration, $\%$ & $3.1(6.4)$ & $7.8(13.7)$ & -4.8 & -12.7 & $0.01 *$ & $<0.05$ \\
\hline Time-weighted average of hypotension, $\mathrm{mmHg}$ & $0.12(0.35)$ & $0.37(1.11)$ & -0.26 & -0.85 & $-0.01 *$ & $<0.05$ \\
\hline
\end{tabular}

CI: confidence Interval; IQR: interquartile range. 
The median time-weighted average of hypotension was $0.12 \mathrm{mmHg}(\mathrm{IQR}, 0.35 \mathrm{mmHg})$ in the intervention group and $0.37 \mathrm{mmHg}(1.11 \mathrm{mmHg})$ in the control group, with a median difference of $-0.25 \mathrm{mmHg}(95 \% \mathrm{Cl}-0.85,-0.01 ; \mathrm{P}<0.05$; Table 2$)$. All patients survived and following our telephone interview, only one patient in the control group referred hospital readmission because of atrial fibrillation. No other complications were referred for the intervention group.

\section{Biochemical markers of organ injury and oxidative stress}

Biochemical markers of organ injury and oxidative stress confirmed to a various extent a correlation with hypotension-related outcomes (Table 3). Specifically, NGAL was correlated with time-weighted average of hypotension $(R=0.316 ; P<0.05)$, and $S 100 B$ protein was correlated with all the primary outcomes investigated (episodes of hypotension, $R=0.584 ; \mathrm{P}<0.001$; absolute time spent in hypotension, $\mathrm{R}=0.628$; $\mathrm{P}<0.001$; time spent in hypotension relative to surgical duration, $R=0.612 ; P<0.001$; time-weighted average of hypotension, $R=0.575 ; P<0.001$ ).

Table 3

Correlation between biochemical markers of inflammation and hypotension-related outcomes.

\begin{tabular}{|c|c|c|c|c|c|c|c|c|c|c|c|c|}
\hline & NGAL & NSE & $\begin{array}{l}\text { HIF - } \\
\text { 1alpha }\end{array}$ & $\begin{array}{l}\text { S100 } \\
\text { B }\end{array}$ & $\begin{array}{l}\text { Acetyl } \\
\text { - CoA }\end{array}$ & $\begin{array}{l}\text { hs } \\
\text { Cardiac } \\
\text { Troponin }\end{array}$ & $\mathrm{LOOH}$ & GSH & $\begin{array}{l}\text { Hypotensive } \\
\text { Episodes }\end{array}$ & $\begin{array}{l}\text { Absolute } \\
\text { time of } \\
\text { hypotension }\end{array}$ & $\begin{array}{l}\text { Relative } \\
\text { time in } \\
\text { hypotension }\end{array}$ & $\begin{array}{l}\text { Time- } \\
\text { weighted } \\
\text { average of } \\
\text { hypotension }\end{array}$ \\
\hline NGAL & 1.000 & - & - & - & - & - & - & - & - & - & - & - \\
\hline NSE & .238 & 1.000 & - & - & - & - & - & - & - & - & - & - \\
\hline $\begin{array}{l}\text { HIF - } \\
\text { 1alpha }\end{array}$ & -.284 & $-.369^{*}$ & 1.000 & - & - & - & - & - & - & - & - & - \\
\hline S100 B & .036 & .258 & -.022 & 1.000 & - & - & - & - & - & - & - & - \\
\hline Acetyl-CoA & $-.540^{\star \star}$ & -.241 & $.426^{\star \star}$ & .123 & 1.000 & - & - & - & - & - & - & - \\
\hline $\begin{array}{l}\text { hs Cardiac } \\
\text { Troponin }\end{array}$ & $.356^{*}$ & $.337^{*}$ & -.304 & .030 & $-.391^{*}$ & 1.000 & - & - & - & - & - & - \\
\hline $\mathrm{LOOH}$ & -.136 & -.103 & -.040 & -.258 & -.010 & -.040 & 1.000 & - & - & - & - & - \\
\hline GSH & .041 & -.213 & .253 & -.283 & .113 & $-.398^{\star \star}$ & -.099 & 1.000 & - & - & - & - \\
\hline $\begin{array}{l}\text { Hypotensive } \\
\text { Episodes }\end{array}$ & .181 & .207 & -.104 & $.584^{\star \star}$ & -.027 & .088 & -.133 & -.132 & 1.000 & & - & - \\
\hline $\begin{array}{l}\text { Absolute } \\
\text { time of } \\
\text { hypotension }\end{array}$ & .288 & .159 & -.113 & $.628^{\star \star}$ & -.111 & .138 & -.121 & -.164 & $.883^{\star \star}$ & 1.000 & - & - \\
\hline $\begin{array}{l}\text { Relative } \\
\text { time in } \\
\text { hypotension }\end{array}$ & .278 & .118 & -.148 & $.612^{* \star}$ & -.096 & .127 & -.199 & -.179 & $.880^{\star \star}$ & $.977^{\star \star}$ & 1.000 & - \\
\hline $\begin{array}{l}\text { Time- } \\
\text { weighted } \\
\text { average of } \\
\text { hypotension }\end{array}$ & $.316^{*}$ & .093 & -.150 & $.575^{\star *}$ & -.153 & .174 & -.160 & -.170 & $.820^{\star \star}$ & $.966^{* *}$ & $.977^{\star *}$ & 1.000 \\
\hline
\end{tabular}

* $p<0.05$ (2-tailed) Mann-Whitney U test; ** $p<0.001$ (2 tailed), Mann-Whitney U test. HIF: hypoxia inducible factor; GSH: reduced glutathione; LOOH: lipid hydroperoxide; NGAL: neuthrophil gelatinase-associated lipocalin; NSE: neuron-specific enolase.

The median difference of biochemical markers of oxidative stress between intervention and control group revealed that the intervention group had lower NSE (difference: $-0.815,95 \% \mathrm{Cl}:-1.485,-0.229 ; \mathrm{P}<0.05)$ and higher $\mathrm{GSH}$ values $(2.6,95 \% \mathrm{Cl}: 0.9,4.6 ; \mathrm{P}<0.05)$. The difference in S100B protein $(-0.8,95 \% \mathrm{Cl}:-1.2,-0.4$, $\mathrm{P}=0.62$ ) did not reach statistical significance (Table 4). 
Table 4

Differences between pre- and post- surgery of biochemical markers of inflammation in intervention and control groups.

\begin{tabular}{|c|c|c|c|c|c|c|c|c|}
\hline & \multicolumn{2}{|l|}{ Intervention } & \multicolumn{2}{|l|}{ Controls } & \multirow[t]{3}{*}{ Median difference } & \multirow[t]{3}{*}{$95 \% \mathrm{Cl}$} & & \multirow[t]{3}{*}{$P$ value } \\
\hline & Pre & Post & Pre & Post & & & & \\
\hline & Median (IQR) & Median (IQR) & Median ( IQR) & Median (IQR) & & & & \\
\hline NGAL (ng/mL) & $2.1(1.7)$ & $2.2(1.8)$ & $2.2(1.4)$ & $2.0(1.0)$ & 0.24 & -0.27 & 0.84 & ns \\
\hline NSE (ng/mL) & $2.0(1.3)$ & $1.0(1.0)$ & $1.9(1.9)$ & $1.7(1.8)$ & -0.81 & -1.48 & -0.23 & $<0.05$ \\
\hline HIF - 1 alpha (ng/mL) & $0.18(0.12)$ & $0.15(0.05)$ & $0.16(0.11)$ & $0.15(0.04)$ & -0.003 & -0.02 & 0.02 & ns \\
\hline $\mathrm{S} 100 \mathrm{~B}(\mathrm{pg} / \mathrm{mL})$ & $0.36(0.34)$ & $0.51(0.63)$ & $0.32(2.0)$ & $1.36(1.29)$ & -0.76 & -1.21 & -0.42 & ns \\
\hline Acetyl - CoA (pmol/ul) & $0.11(0.23)$ & $0.30(1.4)$ & $0.16(0.48)$ & $0.35(2.0)$ & -0.09 & -0.84 & 0.36 & ns \\
\hline Human Cardiac Troponin 1 (pg/mL) & $41.8(32.7)$ & $34.9(36.9)$ & $32.1(36.3)$ & $39.9(39.2)$ & -4.58 & -31.2 & 4.7 & ns \\
\hline $\mathrm{LOOH}$ (nmol/ul) & $6.1(7.6)$ & $4.4(7.1)$ & $4.4(5.0)$ & $3.9(4.8)$ & -0.12 & -3.1 & 2.9 & ns \\
\hline GSH (nmol/ul) & $7.5(7.0)$ & $7.0(3.7)$ & $7.0(5.5)$ & $5.0(2.5)$ & 2.62 & 0.89 & 4.61 & $<0.05$ \\
\hline
\end{tabular}

HIF: hypoxia inducible factor; IQR: interquartile Range; GSH: reduced glutathione; LOOH: lipid hydroperoxide; NGAL: neuthrophil gelatinase-associated lipocalin; NSE: neuron-specific enolase. Differences were evaluated with Mann-Whitney U test. ns: not significant

\section{Discussion}

Our pilot study investigated the ability of a machine learning-derived EWS in combination with an algorithm for hemodynamic management in reducing hypotension episodes and duration during major general surgery. Furthermore, we evaluated the clinical impact of intraoperative hypotension on end-organ damage and oxidative stress with a series of biochemical assays.

We found that the intervention group had significantly reduced incidence and duration of intraoperative hypotension, as well as lower time-weighted average of hypotension during surgery. Our results are consistent with previous studies showing that the EWS was able to predict hypotension with good sensitivity and specificity[10,11]. To the best of our knowledge, our study is the first one demonstrating that the use of an EWS with an hemodynamic algorithm with the consequent reduction in intraoperative hypotension was associated also with a significant reduction in the value of biomarkers of organ injury and oxidative stress.

Our results add knowledge to the growing body of evidence regarding the role of HPI in predicting intraoperative episodes of hypotension, thus allowing a proactive anesthesiological approach. Once an alarm was detected (HPI>85\%), the anesthesiologist in charge followed a treatment algorithm based on advanced hemodynamic parameters, which suggested vasopressor, fluid and/or inotrope administration (alone or in combination), or eventually observation. We found a significant reduction in hypotension in the intervention group and this was consistent with all the measures performed (episodes, absolute and relative time of hypotension, and time-weighted average). The time-weighted average of hypotension seems a very promising variable to evaluate the degree of intraoperative hypotension, taking into account both the severity of hypotension and its duration as well as the total surgical time. In this regards, our intervention group found similar values of time-weighted average $(0.12 \mathrm{mmHg})$ as compared to other larger studies $(0.10 \mathrm{mmHg}[10] \mathrm{and} 0.14 \mathrm{mmHg}[12])$. Indeed, our results seem in line with most of currently published findings. Although a recent study by Maheshwari et al conducted in 214 patients undergoing non-cardiac surgery questioned the role of HPI[12], most of the published evidence supports the value of HPI in predicting hypotension. Indeed, such results have been found in patients undergoing total hip arthroplasty[13], major/general surgery[14, 15] and cardiac surgery[16]. Such studies have demonstrated good sensibility and specificity in predicting hypotension from 5 minutes (both around $85 \%$ ) to 15 minutes (both around $75 \%-80 \%$ ) before the hypotensive episode[14, 16, 17]. Of note, two of these studies evaluated the use of HPI throughout non-invasive arterial pressure waveform, with encouraging findings[15, 17].

Our study has the originality of evaluating also biochemical aspects, thus functioning as a pilot study for the investigation of the impact of intraoperative hypotension on oxidative stress and end-organ injury. Of course, the reliability of our findings are limited by the small sample size, but the correlation between the primary outcome measures and several biomarkers may warrant further investigation.

Firstly, we showed that HIF-1a is not significantly up-regulated in both groups and is not dependent on the number and duration of hypotensive events. We hypothesized that one possible complication of hypoxia could be recurrent hypoxia which causes modifications of gene transcription as well as posttranslational protein modification, including the ones regulating metabolism or cardiovascular system. HIF is a heterodimeric complex, which consists of two subunits: $a(H I F ~ a)$ and $\beta$ (HIF $\beta$ ) [18]. Subunit $a$ is oxygen-sensitive, during normoxia is associated with von Hippel-Lindau (VHL) protein, which is responsible for the induction of its proteasome degradation[19]. Therefore, in normoxia, the half lifetime of HIF1a is greatly shorted than in hypoxia conditions [20], as low pressure of oxygen is responsible for blocking the binding of VHL and HIF1a and its degradation is then inhibited [21]. Recently, Gabryelska A et al. [22] showed that serum HIF-1 a was higher in obstructive sleep apnea patients when compared to control. In particular, control groups exhibited HIF-1a serum levels comparable to both groups included in our studies whereas obstructive sleep apnea patients showed much higher values. However, it should be noted that HIF-1 $\mathrm{a}$ is generally up-regulated during chronic hypoxic conditions and therefore it is conceivable that hypotension, despite causing a possible oxygen 
supply-demand mismatch, is not sufficient in terms of duration and oxygen delivery to trigger the HIF-1a pathway. This hypothesis is consistent with our data showing non-significant changes in lactate and acetyl-CoA levels in both groups of patients, thus suggesting that cells are not metabolically rewiring toward an hypoxic phenotype. To the best of our knowledge this is the first report evaluating the possible activation of HIF-1a pathway activation during hypotension. On the other hand, we observed a significant reduction of the reduced form of GSH in the controls when compared to the intervention group, thus suggesting that hypotensive episodes increased oxidative stress and that the application of a machine learning-derived EWS for pending intraoperative hypotension in combination with a hemodynamic diagnostic guidance may be sufficient in preventing such condition. These results are also consistent with previous reports showing that oxidative stress is a variable and common condition occurring during surgical procedures [22]; however, other variables (i.e.

ischemia/reperfusion, surgical procedures, anesthesia protocols) rather than hypotension have been advocate in order to explain intraoperative oxidative stress. Interestingly, non-significant changes were observed for lipid peroxidation markers. Two non-mutually exclusive hypotheses may be responsible to explain such result. The first is that the reduced GSH and its enzymatic biosynthetic machinery is sufficient to counteract the production of lipid hydroperoxides. To this regard, we should note that we excluded from the study those patients affected by chronic diseases which are also responsible for imbalanced glutathione synthesis (i.e. liver diseases). The second possibility is that other more instable adducts are produced downstream the lipid peroxidation pathway (i.e. hydroxynonenal, malonyldhyaldheyde, etc) and are therefore not detected by the used analysis in the present study. Furthermore, NGAL and troponin were not significant in the intervention group when compared to controls. Interestingly, we also observed a significant reduction of NSE in the intervention group when compared to control. This result is of particular relevance since no previous clinical studies reported neurological complications following intraoperative hypotensive events. Furthermore, increased circulating NSE levels seems to be specific of intraoperative hypotensive events since previous reports showed that NSE was not significantly changed following hypotension in cardiac arrest patients [23]. However, it should be noted that hypotensive events during intraoperative procedures are clinically different from those occurring during cardiac arrest in terms of number of episodes and duration. On the other hand, increased NSE levels, but not S100B, were observed in patients undergoing controlled hypotension during skull base procedures [24]. These results are also consistent with our observations regarding S100B levels. To this regard, even though S100B did not reach statistical significance it showed a trend similar to NSE. It is possible that an increased sample size would clarify this issue. Finally, previous reports showed that both biomarkers of brain injury can cross blood-brain barrier with different intensity, irrespective of differences in their molecular weight (NSE>S100B) [25]. However, it remains to be determined whether increased NSE levels are dependent on increased troponin levels following cardiac impairment or it is a direct consequence of increased oxidative stress mediators formed outside the central nervous system. Studies using near infrared spectroscopy associated with intraoperative events monitoring are currently running in our unit in order to further elucidate this point. Finally, our study did not include a long-term neurological examination of enrolled patients and therefore it is not possible to determine whether increased NSE levels are also associated to the clinical outcome in the postoperative period.

Taken all together, our study confirms the importance of monitoring and preventing intraoperative hypotension, reinforcing the clinically meaningful impact that its occurrence has on end-organ damage and oxidative stress. Future multicenter studies on a larger cohort of patients are now warranted in order to fully elucidate the clinical effectiveness of hypotension prevention on organ injury.

\section{Limitations}

Our study has several limitations that should be considered. First, it is a single center study in a relatively small sample size. Larger and multicenter studies are warranted to support or not our findings. Second, the study was not powered enough to investigate the correlation of intraoperative hypotension with short and long-term postoperative complications. Third, it remains to be studied if the higher values of several biomarkers of organ injury and oxidative stress truly correlates with postoperative organ damage and with patient's outcome. Forth, we included a heterogeneous sample of patients undergoing major noncardiac surgery and with different perioperative risk stratification. Considering the volume of surgery at our Institution it would have not been feasible to enroll patients undergoing the same surgical operation.

\section{Conclusions}

The use of an EWS monitoring on the risk of hypotension coupled with a hemodynamic algorithm significantly reduced the occurrence and duration of intraoperative hypotension. Patients randomized to EWS monitoring had lower postoperative values of several biomarkers of organ injury and oxidative stress.

\section{Declarations}

Ethics approval and consent to participate: The study was approved by the local institutional review board (reference protocol number: NL62115.018.17). Informed consent was obtained in written form from all the patients enrolled

Ethics accordance statement: all methods were performed in accordance with the relevant guidelines and regulations.

Consent for publication: All authors give their consent to publication of the present article

Availability of data and materials: The datasets used and/or analyzed during the current study are available from the corresponding author on reasonable request

Competing interests: The authors declare no competing interests

Funding Statement: This work was supported in part by a liberal and non-conditioned donation of Edwards LifeScience to Prof. Paolo Murabito and by a grant from the University of Catania (Piano Piaceri 2019-2022). 
Acknowledgements: None

Conflicts of Interest: The authors declare no conflict of interest

\section{AUTHORS CONTRIBUTION}

P.M. and G.L.V. conceived and designed the study, L.L.V., L.L., F.V. and A.D. acquired the data, M.A., F.B. and A.C. analyzed and interpreted the data, P.M. and G.L.V. drafted the article, F.S. revised it critically for important intellectual content, all authors finally approved the version to be submitted.

\section{References}

1. Walsh M, Devereaux PJ, Garg AX, Kurz A, Turan A, Rodseth RN, Cywinski J, Thabane L, Sessler DI: Relationship between intraoperative mean arterial pressure and clinical outcomes after noncardiac surgery: toward an empirical definition of hypotension. Anesthesiology 2013, 119(3):507-515.

2. Monk TG, Bronsert MR, Henderson WG, Mangione MP, Sum-Ping ST, Bentt DR, Nguyen JD, Richman JS, Meguid RA, Hammermeister KE: Association between Intraoperative Hypotension and Hypertension and 30-day Postoperative Mortality in Noncardiac Surgery. Anesthesiology 2015, 123(2):307-319.

3. Sun LY, Wijeysundera DN, Tait GA, Beattie WS: Association of intraoperative hypotension with acute kidney injury after elective noncardiac surgery Anesthesiology 2015, 123(3):515-523.

4. van Waes JA, van Klei WA, Wijeysundera DN, van Wolfswinkel L, Lindsay TF, Beattie WS: Association between Intraoperative Hypotension and Myocardial Injury after Vascular Surgery. Anesthesiology 2016, 124(1):35-44.

5. Bijker JB, van Klei WA, Kappen TH, van Wolfswinkel L, Moons KG, Kalkman CJ: Incidence of intraoperative hypotension as a function of the chosen definition: literature definitions applied to a retrospective cohort using automated data collection. Anesthesiology 2007, 107(2):213-220.

6. Botto F, Alonso-Coello P, Chan MT, Villar JC, Xavier D, Srinathan S, Guyatt G, Cruz P, Graham M, Wang CY et al: Myocardial injury after noncardiac surgery: a large, international, prospective cohort study establishing diagnostic criteria, characteristics, predictors, and 30-day outcomes. Anesthesiology 2014 , 120(3):564-578.

7. Landesberg G, Mosseri M, Shatz V, Akopnik I, Bocher M, Mayer M, Anner H, Berlatzky Y, Weissman C: Cardiac troponin after major vascular surgery: the role of perioperative ischemia, preoperative thallium scanning, and coronary revascularization. J Am Coll Cardiol 2004, 44(3):569-575.

8. Thygesen K, Alpert JS, Jaffe AS, Simoons ML, Chaitman BR, White HD, Joint ESCAAHAWHFTFfUDoMI, Authors/Task Force Members C, Thygesen K, Alpert JS et al: Third universal definition of myocardial infarction. J Am Coll Cardiol 2012, 60(16):1581-1598.

9. Vernooij LM, van Klei WA, Machina M, Pasma W, Beattie WS, Peelen LM: Different methods of modelling intraoperative hypotension and their association with postoperative complications in patients undergoing non-cardiac surgery. British journal of anaesthesia 2018, 120(5):1080-1089.

10. Wijnberge M, Geerts BF, Hol L, Lemmers N, Mulder MP, Berge P, Schenk J, Terwindt LE, Hollmann MW, Vlaar AP et al: Effect of a Machine Learning-Derived Early Waming System for Intraoperative Hypotension vs Standard Care on Depth and Duration of Intraoperative Hypotension During Elective Noncardiac Surgery: The HYPE Randomized Clinical Trial. Jama 2020, 323(11):1052-1060.

11. Hatib F, Jian Z, Buddi S, Lee C, Settels J, Sibert K, Rinehart J, Cannesson M: Machine-learning Algorithm to Predict Hypotension Based on High-fidelity Arterial Pressure Waveform Analysis. Anesthesiology 2018, 129(4):663-674.

12. Maheshwari K, Shimada T, Yang D, Khanna S, Cywinski JB, Irefin SA, Ayad S, Turan A, Ruetzler K, Qiu Y et al: Hypotension Prediction Index for Prevention of Hypotension during Moderate- to High-risk Noncardiac Surgery. Anesthesiology 2020, 133(6):1214-1222.

13. Schneck E, Schulte D, Habig L, Ruhrmann S, Edinger F, Markmann M, Habicher M, Rickert M, Koch C, Sander M: Hypotension Prediction Index based protocolized haemodynamic management reduces the incidence and duration of intraoperative hypotension in primary total hip arthroplasty: a single centre feasibility randomised blinded prospective interventional trial. Journal of clinical monitoring and computing 2020, 34(6):1149-1158.

14. Davies SJ, Vistisen ST, Jian Z, Hatib F, Scheeren TWL: Ability of an Arterial Waveform Analysis-Derived Hypotension Prediction Index to Predict Future Hypotensive Events in Surgical Patients. Anesthesia and analgesia 2020, 130(2):352-359.

15. Wijnberge M, van der Ster BJP, Geerts BF, de Beer F, Beurskens C, Emal D, Hollmann MW, Vlaar APJ, Veelo DP: Clinical performance of a machine-learning algorithm to predict intra-operative hypotension with noninvasive arterial pressure waveforms: A cohort study. European journal of anaesthesiology 2021, 38(6):609-615

16. Shin B, Maler SA, Reddy K, Fleming NW: Use of the Hypotension Prediction Index During Cardiac Surgery. J Cardiothorac Vasc Anesth 2021, 35(6):17691775.

17. Maheshwari K, Buddi S, Jian Z, Settels J, Shimada T, Cohen B, Sessler DI, Hatib F: Performance of the Hypotension Prediction Index with non-invasive arterial pressure waveforms in non-cardiac surgical patients. Journal of clinical monitoring and computing 2021, 35(1):71-78.

18. Semenza GL: Hypoxia-inducible factors in physiology and medicine. Cel/ 2012, 148(3):399-408.

19. Kaelin WG, Jr., Ratcliffe PJ: Oxygen sensing by metazoans: the central role of the HIF hydroxylase pathway. Mo/ Cel/ 2008, 30(4):393-402

20. Berra E, Roux D, Richard DE, Pouyssegur J: Hypoxia-inducible factor-1 alpha (HIF-1 alpha) escapes O(2)-driven proteasomal degradation irrespective of its subcellular localization: nucleus or cytoplasm. EMBO Rep 2001, 2(7):615-620.

21. Epstein AC, Gleadle JM, McNeill LA, Hewitson KS, O'Rourke J, Mole DR, Mukherji M, Metzen E, Wilson MI, Dhanda A et al: C. elegans EGL-9 and mammalian homologs define a family of dioxygenases that regulate HIF by prolyl hydroxylation. Cell 2001, 107(1):43-54.

22. Gabryelska A, Szmyd B, Szemraj J, Stawski R, Sochal M, Bialasiewicz P: Patients with obstructive sleep apnea present with chronic upregulation of serum HIF-1alpha protein. J Clin Sleep Med 2020, 16(10):1761-1768. 
23. Jakkula P, Pettila V, Skrifvars MB, Hastbacka J, Loisa P, Tiainen M, Wilkman E, Toppila J, Koskue T, Bendel S et al: Targeting low-normal or high-normal mean arterial pressure after cardiac arrest and resuscitation: a randomised pilot trial. Intensive care medicine 2018, 44(12):2091-2101.

24. Sieskiewicz A, Groblewska M, Lyson T, Piszczatowski B, Turek G, Rutkowski R, Mroczko B, Rogowski M, Mariak Z: Neurobiochemical markers of brain ischemia in patients subjected to endoscopic skull base surgery under controlled hypotension. J Neurosurg Sci 2016, 60(3):313-319.

25. Pfeifer R, Borner A, Krack A, Sigusch HH, Surber R, Figulla HR: Outcome after cardiac arrest: predictive values and limitations of the neuroproteins neuronspecific enolase and protein S-100 and the Glasgow Coma Scale. Resuscitation 2005, 65(1):49-55.

\section{Figures}

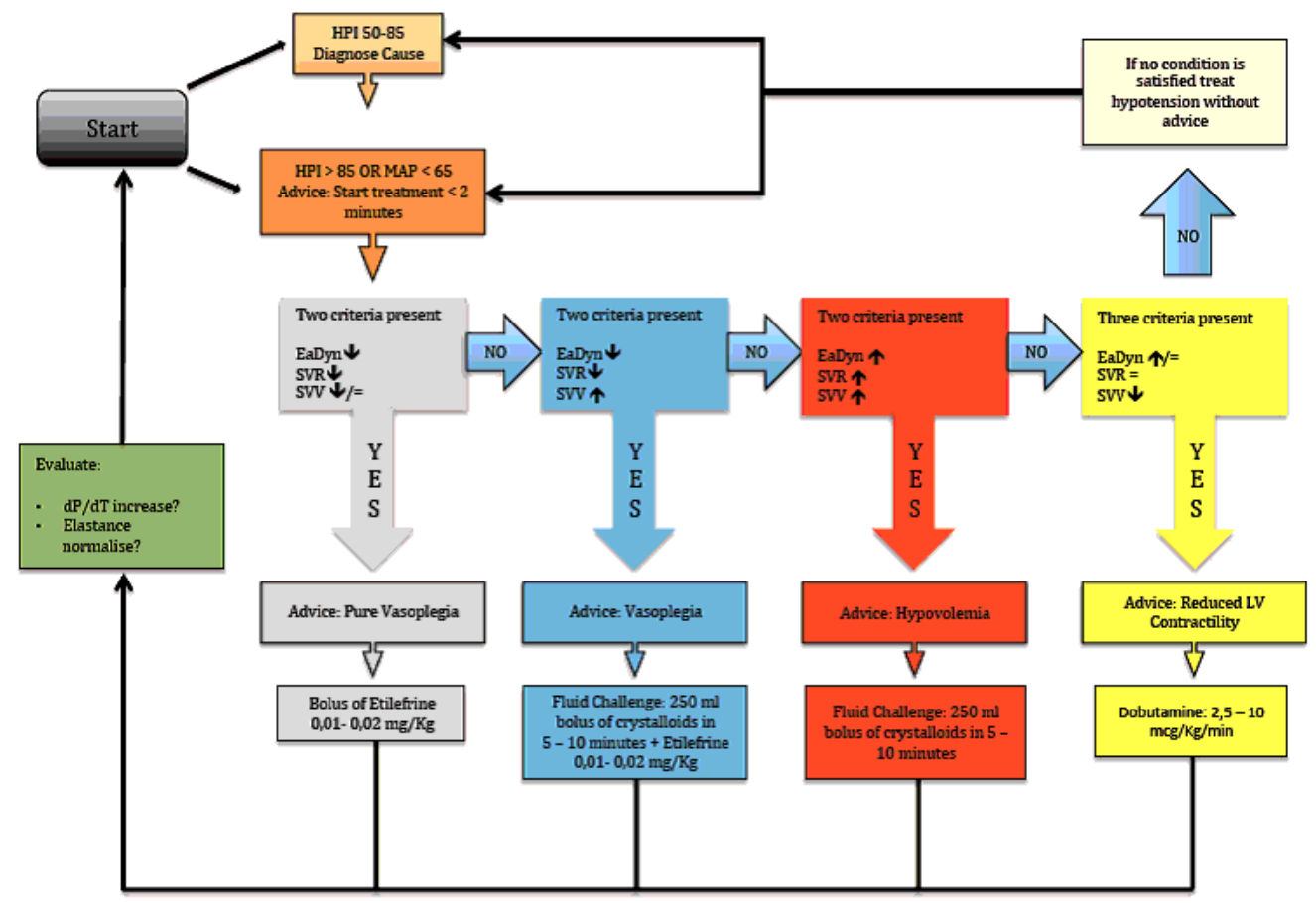

Figure 1

Algorithm for hemodynamic management used in the intervention group and coupled with the hypotension prediction index.

\section{Supplementary Files}

This is a list of supplementary files associated with this preprint. Click to download.

- HPIprotocol.pdf

- Supplementarymaterial3.docx

- CONSORT2010Checklist.doc 